\title{
Neurotrophic Effects of Electroconvulsive Therapy: A Proton Magnetic Resonance Study of the Left Amygdalar Region in Patients with Treatment-Resistant Depression
}

\author{
Nikolaus Michael*,', Andreas Erfurth', Patricia Ohrmann', Volker Arolt', Walter Heindel ${ }^{2}$ and Bettina \\ Pfleiderer ${ }^{2}$ \\ 'Department of Psychiatry, University of Münster, Münster, Germany; ${ }^{2}$ Department of Clinical Radiology, University of Muenster, Münster, \\ Germany
}

\begin{abstract}
Negatively balanced neurotrophic factors may be important in precipitating clinical depression. Recently, it has been reported that antidepressant therapy may exert positive neurotrophic effects. The aim of this study was to detect probable neurotrophic changes during electroconvulsive therapy (ECT). For this purpose, $\mathrm{N}$-acetylaspartate (NAA), an amino acid exclusively located in neurons, and other brain metabolites such as glutamine/glutamate (Glx), choline (Cho), and creatine ( $\mathrm{Cr}$ ) were measured in patients by localized proton magnetic resonance spectroscopy. A total of 28 severely depressed patients (DSM-IV) were enrolled, and the left amygdalar region was investigated by proton STEAM spectroscopy before and after unilateral ECT. The results were compared with 28 age- and gender-matched controls using nonparametric paired and unpaired tests. A significant increase in NAA was observed only in ECT responders $(n=14 ; p=0.019)$. Five out of 14 nonresponders to ECT monotherapy were remeasured following a clinical improvement after continued ECT combined with antidepressants and were then found also to present a significant increase in NAA. In all successfully treated patients, parallel observations, that is, increased levels, were made for $\mathrm{Gl}$, whereas $\mathrm{Cho}$ and $\mathrm{Cr}$ were unchanged. In conclusion, our preliminary finding of increased NAA concentrations after successful ECT may indicate a probable neurotrophic effect of ECT. Neuropsychopharmacology (2003) 28, 720-725. doi:I0.I038/sj.npp. I 300085
\end{abstract}

Keywords: electroconvulsive therapy; depression; neurotrophic effects; proton magnetic resonance spectroscopy

\section{INTRODUCTION}

Electroconvulsive therapy (ECT) is well known for its strong antidepressant effect, but there is little information about the neurobiological mechanisms underlying these effects (Fink, 2001). Recent developments in cell and molecular biology have led to the hypothesis that reductions in neurotrophic signaling may be important in precipitating clinical depression (Duman and Vaidya, 1998; Jacobs et al, 2000). There is considerable evidence that antidepressant therapy exerts positive neurotrophic effects (Manji et al, 2000). Since it was shown in animal experiments that ECT induces mossy fiber sprouting in the rat hippocampus (Chen et al, 2000; Lamont et al, 2001; Vaidya et al, 1999) and stimulates neurogenesis (Madsen et al, 1998), a neurotrophic mode of antidepressive action was also proposed for ECT (Duman and Vaidya, 1998). However,

*Correspondence: Dr $N$ Michael, Department of Psychiatry, University of Münster, Albert-Schweitzer-Str. II, 48129 Münster, Germany, Tel: +49 25I 83 56626, Fax: +49 25I 83 56612, E-mail: nikmich@uni-muenster.de

Received 17 April 2002; revised 8 August 2002; accepted 3 October 2002

Online publication: 10 October 2002 at http://www.acnp.org/citations/ Npp I01002405 neurotrophic effects are difficult to detect in the human brain in vivo. Proton magnetic resonance spectroscopy $\left({ }^{1} \mathrm{H}\right.$ MRS) poses an unprecedented opportunity for noninvasive assessment of brain metabolism in well-defined brain areas. $\mathrm{N}$-acetylaspartate (NAA), an amino acid synthesized primarily in neurons (Simmons et al, 1991) and detectable by MRS, can be used as an indirect measure of neuron functionality (Sager et al, 2001; Tsai et al, 1995). Moreover, mature oligodentrocytes can express NAA in vitro (Bhakoo and Pearce, 2000) and it was suggested that glia cells are also involved in NAA/NAAG metabolism. NAA/NAAG may play an important role in cell-specific glia signaling and in the establishment and maintenance of the nervous system (Baslow, 2000). Recently, NAA increase was observed after long-term lithium therapy in cortical brain areas of bipolar patients (BIPs) (Moore et al, 2000), indicating a neurotrophic/neuroprotective effect. A recent ${ }^{1} \mathrm{H}$ MRS study investigating bilateral hippocampi of ECT-treated patients found NAA levels unaltered (Ende et al, 2000).

There is some evidence to be derived from morphological (Ali et al, 2001; Altshuler et al, 1998), neuropathological (Sheline et al, 1998), and functional imaging studies (Drevets, 2000) that the amygdalar region is involved in the pathophysiology of depression. Here, we investigated 28 severely depressed patients by ${ }^{1} \mathrm{H}$ MRS before and after ECT 
to assess probable neurotrophic and other metabolic effects related to ECT. We chose a voxel comprising the left amygdala, including the anterior hippocampus, a region centrally involved in the regulation of emotion and vigilance (Davis and Whalen, 2001).

\section{MATERIALS AND METHODS}

\section{Patients}

A total of 28 patients fulfilling the diagnostic criteria for major depressive episode (DSM-IV) and referred to ECT because of treatment resistance were enrolled in this study. Patients were classified as being severely depressed due to their high Montgomery-Asperg-rating scale (MADRS) values and length of index episode (Table 1). The diagnosis was made independently by two experienced psychiatrists employing the Munich checklist for DSM-IV diagnoses (Hiller et al, 2000). Patients with a medical history of alcohol and drug abuse or dementia were excluded. Treatment resistance was defined as failure to respond to at least two different antidepressants given longer than 4 weeks at the maximum recommended dose. All patients were off psychotropic medication between 3 and 8 days (Table 1) (only lorazepam allowed at maximum $3 \mathrm{mg} /$ day) and were investigated before and after ECT. The reason for discontinuation of psychotropic medication was to provide a standard ECT monotherapy protocol; however a longer washout period was not feasible, since our patients were severely ill and a longer postponing of indicated ECT was not acceptable. After a detailed description of the study to the subjects, written informed consent was obtained. The protocol was approved by the university ethics committee and the studies were carried out in accordance with the Declaration of Helsinki. The sample consisted of 13 unipolar (UPs) and 15 BIPs. Clinical characteristics are summarized in Table 1; there was no statistically significant intergroup difference regarding relevant clinical parameters. Response was defined as a MADRS reduction
$>60 \%$ of baseline scores (Prudic et al, 1996). Patients with an insufficient clinical response were put on combined ECT/pharmacotherapy. After cessation of ECT monotherapy, 14 patients had not responded; five of these were remeasured after finally responding. Pharmacotherapy was different for each patient (patient 1: trijod-thyronine, venlafaxine; patient 2: lithium; patient 3: lithium, valproic acid; patient 4: tranylcypromine, valproic acid; patient 5: tranylcypromine, lithium). The number of additional ECTs was $8(n=1)$ for the UPs and $7.3 \pm 7.2$ $(n=4)$ for the BIPs.

Age- and gender-matched healthy controls (unipolar controls: mean age $58.5 \pm 8.1$ years; 9 female; bipolar controls: mean age $52.2 \pm 14.4$ years; 10 female) were enrolled. Written informed consent was obtained and the same standardized interview as for patients was performed to rule out any underlying psychiatric or neurologic condition (Hiller et al, 2000).

\section{ECT Treatment}

ECT was applied using a customized brief-pulse device (Thymatron IV, Somatics, Lake Bluff, IL, USA) beginning with stimulus titration in the first session and continuing with the 2.5-fold stimulus dose. Motor and EEG seizure duration were monitored, and conservative criteria for adequacy were used, with the stimulus intensity being adjusted accordingly. Methohexital $(0.75-1.5 \mathrm{mg} / \mathrm{kg})$ and succinylcholine $(0.5-1.0 \mathrm{mg} / \mathrm{kg})$ were applied for anesthesia and relaxation. No atropine was applied prior to the treatment. Patients required a mean of $11.6 \pm 5$ ECT treatments. In 27 of the 28 patients stimulation was right unilateral, and in one bilateral.

\section{MRS Measurements}

The MRI protocol included T1-weighted 3D spoiled gradient echo acquisition of the whole brain and T2- and proton density-weighted fast spin echo sequences in

Table I Clinical Characteristics of Patient and Control Samples

\begin{tabular}{|c|c|c|}
\hline & $\begin{array}{l}\text { Unipolar depressives } \\
\qquad(n=13)\end{array}$ & $\begin{array}{l}\text { Bipolar depressives } \\
\qquad(n=15)\end{array}$ \\
\hline Age (years) & $\begin{array}{l}59.7 \pm 15.2 \\
58.5 \pm 8.9 \text { (controls) }\end{array}$ & $\begin{array}{l}54.1 \pm 16 \\
52.2 \pm 14.4 \text { (controls) }\end{array}$ \\
\hline Gender & Nine females & Nine females \\
\hline Diagnosis & & Seven BIP I, seven BIP II; one BIP \\
\hline Duration of illness (years) & $15.2 \pm 11.5$ & $19.9 \pm 12.2$ \\
\hline Number of hospitalizations & $3.8 \pm 2.9$ & $3.3 \pm 2$ \\
\hline Number of depressive episodes & $5.2 \pm 5.5$ & $4.7 \pm 3.7$ \\
\hline Duration of index episode (months) & $14.3 \pm 11.1$ & $20.9 \pm 23.6$ \\
\hline Lithium pre-ECT & $n=2(15.4 \%)$ & $n=4(26.6 \%)$ \\
\hline Arterial hypertension & $n=2(15.4 \%)$ & $n=5(33 \%)$ \\
\hline Off-medication period (days) & $4.2 \pm 2.1$ & $7.7 \pm 6.2$ \\
\hline Number of sufficient antidepressant trials in index episode ${ }^{b}$ & $2.6 \pm 1$ & $2.9 \pm 1.2$ \\
\hline Pre-ECT: MADRS & $36.7 \pm 7.5$ & $34.9 \pm 7$ \\
\hline Post-ECT monotherapy: MADRS & $\begin{array}{c}8 \pm 5.2(n=10 ; \text { responders }) \\
26 \pm 7.2(n=3 ; \text { nonresponders })\end{array}$ & $\begin{array}{c}6.2 \pm 4.4(n=4 ; \text { responders }) \\
22.8 \pm 6(n=11 ; \text { nonresponders })\end{array}$ \\
\hline Number of ECTs ${ }^{c}$ & $\begin{array}{l}8.7 \pm 3.1 ; \text { nonresponders } \\
9.8 \pm 2.8 ; \text { responders }\end{array}$ & $\begin{array}{l}9 \pm 2.5 ; \text { nonresponders } \\
8 \pm 2 ; \text { responders }\end{array}$ \\
\hline
\end{tabular}

${ }^{\mathrm{a} B i p o l a r}$ not otherwise specified. ${ }^{\mathrm{b}}$ Maximum recommended dose for at least 4 weeks. ${ }^{\mathrm{C}}$ ffter completion of ECT monotherapy. 
transaxial and coronal orientation. For the MRS measurements, single voxel STEAM spectroscopy $(\mathrm{TE}=20 \mathrm{~ms}$, $\mathrm{TR}=2.5 \mathrm{~s}$, voxel size $(\mathrm{VOI})=3.375 \mathrm{~cm}^{3}$, number of scans $=128$ ) at $1.5 \mathrm{~T}$ (Magnetom SP, Siemens, Erlangen, Germany) was used (voxel position: left amygdalar region-comprising the maximum of amygdalar gray matter (GM) and the anterior one-third of the hippocampus). Voxel positions between scans were referenced to readily identifiable anatomical landmarks (inferior temporal horn).

\section{Postprocessing and Data Analysis}

To account for variations in voxel composition (cerebral spinal fluid (CSF), GM, and white matter), segmentation of the voxels was performed with a semiautomatic interactive algorithm (M Fiebich, Department of Biomedical Engineering, University of Applied Sciences, Giessen, Germany). The metabolite concentrations were corrected for partial volume effects (CSF) and normalized to the GM fraction in the voxels (Wang and Li, 1998) and coil loading. After postprocessing of our spectra, metabolite concentrations were calculated using a time domain fitting program (LC model (Provencher, 1993); Figure 1). Owing to overlapping resonances of glutamate, glutamine, and GABA at a field strength of $1.5 \mathrm{~T}$, only a combined fitting of $\mathrm{Glx}$ (glutamine, glutamate) was performed. GABA was excluded from the processing and data analysis, since the fitting error (percent standard deviation SD) was too high to be meaningful. For robustness of results, only metabolite information with a fitting error of $<20 \%$ SD was included in the final analysis. Contribution of the altered GABA levels may still influence our data. However, the GABA concentration in general is much lower (about $1 \mathrm{mmol} / \mathrm{kg}$ brain tissue (Sanacora et al, 1999)) than combined Glu/ Gln (about 12-14 mmol/kg brain tissue). Also, the Glx signal is mostly dominated by glutamate (Glu) (Auer et al, 2000).

Statistical analysis was performed with SPSS software (SPSS 10.0 for Windows, SPSS Inc., Chicago, Illinois, USA).
The Mann-Whitney test was employed as a nonparametric test between groups, the Wilcoxon test as a paired test for metabolite changes after treatment, and the Pearson test for correlation analysis.

\section{RESULTS}

Before ECT, the concentrations of NAA, choline (Cho), and creatine $(\mathrm{Cr})$ in patients were not statistically different from those of controls. Only Glx showed a trend towards a reduction $(p=0.056)$. Glx reduction was significant only in patients with unipolar depression $(p=0.049)$. Segmentation of the MRS voxels revealed that the GM fraction was significantly higher in patients $(p<0.0001)$ compared to controls. ECT did not affect voxel composition, segmentation before and after ECT did not reveal differences. A positive correlation was found between GM fraction and duration of illness $(p=0.024)$. Successful ECT was accompanied by increased NAA $(p=0.019$, Figure 2) and Glx levels $(p=0.049)$ compared to baseline before ECT for all patients. The number of ECTs did not differ between responders and nonresponders (Table 1). Cho and Cr levels increased in responders without reaching statistical significance.

When analyzing BIPs and UPs separately, the diagnosis had no major influence on our results, with the exception of reduced Glx levels pre-ECT. Only unipolar depressed patients had significantly reduced Glx levels compared to controls $(p=0.04)$.

Five ( $n=4$ BIPs I, $n=1$ UP) of the nonresponding patients were remeasured after they had finally responded to continued ECT combined with pharmacotherapy. Hereafter, they also showed a similar increase in NAA $(p=0.043$; Figure 3).

\section{DISCUSSION}

In this study we investigated a clinically highly important subgroup of severely ill, treatment-resistant patients with major depressive disorder to see whether probable

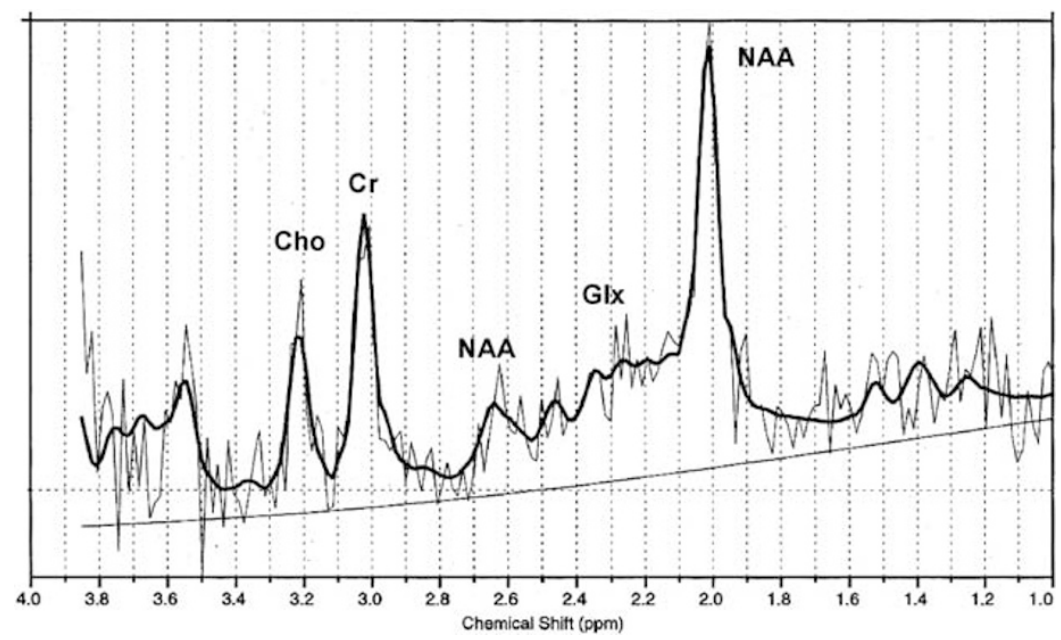

Figure I Original and fitted (LC model, black line) 'H MRS spectra of the left amygdalar region of a 54-year-old female patient pre-ECT. Baseline correction, but no line broadening was applied. $\mathrm{NAA}=\mathrm{N}$-acetylaspartate, $\mathrm{Gl}=$ glutamate, glutamine, $\mathrm{Cr}=\mathrm{creatine}$ and $\mathrm{Cho}=\mathrm{choline-containing}$ compounds. 

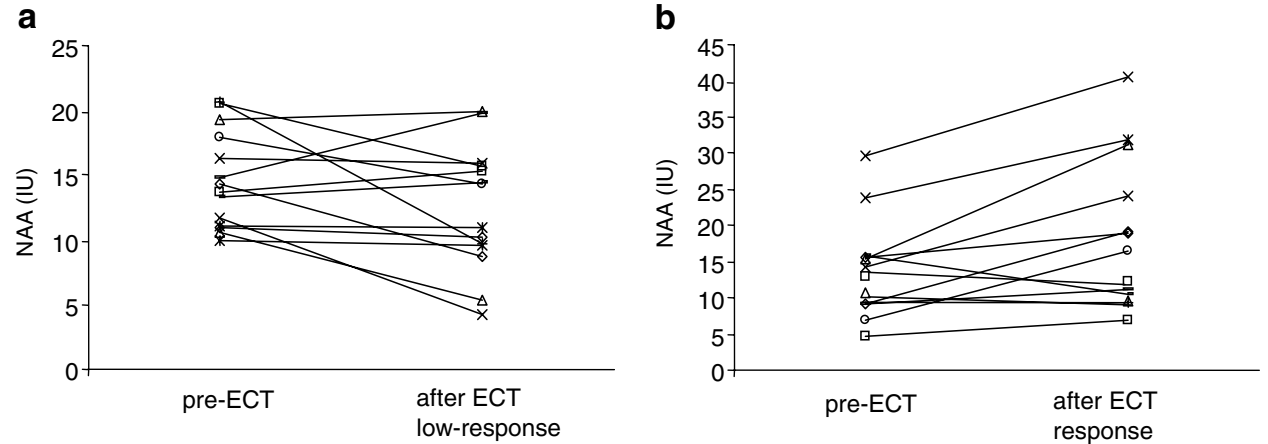

Figure 2 NAA levels (institutional units) before and after ECT monotherapy. (a) In treatment nonresponders ( $n=14)$ NAA did not increase, while (b) in treatment responders $(n=14)$ NAA increased significantly (nonparametric paired test, Wilcoxon, $p=0.019$ ).

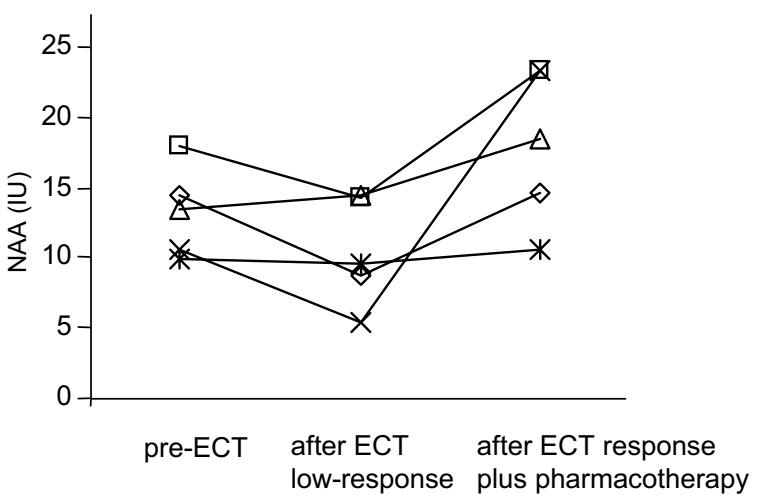

Figure 3 NAA levels (institutional units) during ECT course $(n=5)$. Low NAA levels of ECT nonresponders increased significantly after successful treatment (nonparametric paired test, Wilcoxon, $p=0.043$ ).

neurotrophic effects may play a role in the strong antidepressant efficacy of ECT. There is increasing evidence that negatively balanced neurotrophic factors may contribute to the precipitation of severe mood disorders (Duman et al, 1997; Jacobs et al, 2000). On the other hand, as known from neurobiological studies, antidepressant strategies, for example, tricyclics, lithium, or ECT, may increase neuronal functionality/viability (Duman et al, 1999).

The major finding of this study was that ECT therapy was accompanied by a significant increase in NAA levels in responders (Figure 2). NAA is located exclusively in neurons (Simmons et al, 1991) and is suggested to be a marker of neuronal viability and functionality. It has been reported that chronic lithium therapy in therapeutic doses leads to increased NAA levels because of increased neuronal viability, probably mediated by a neuroprotective protein of B-cell lymphoma/leukemia-2 called bcl-2 (Moore et al, 2000); bcl-2 has been shown to prevent neuronal cell death in vivo and lithium therapy is able to induce its robust increase. The parallel findings of increased NAA levels after ECT as well as after lithium therapy suggest a similar neurotrophic effect.

Additionally, ECT-induced structural changes have been described in animal experiments, that is, mossy fiber/ synaptic sprouting (Lamont et al, 2001; Vaidya et al, 1999) or neurogenesis (Madsen et al, 2000; Parent et al, 1997). Overall, these findings may indicate improved neuronal functioning (Moore et al, 2000).

The missing NAA increase in nonresponders probably indicates that a neurotrophic effect may be important for antidepressant efficacy. Differences between responders and nonresponders to ECT were observed by Bonne et al (1996) by single-photon emission computerized tomography (SPECT), who described reduced cerebral blood flow preECT and an increase only in ECT responders. The most robust changes were observed in the cingulate gyrus, which is part of the paralimbic cortex and also but less pronounced in the parietal lobes.

Five of our 14 nonresponding patients remeasured after successful ECT combined with antidepressants (three of them additionally treated with lithium) presented a similar increase in NAA levels (Figure 3). Since three of five nonresponding patients received lithium and lithium can also increase NAA levels (Moore et al, 2000), we cannot rule out that this increase may be because of the effect of lithium and less to the positive ECT response.

Although the number of patients in the follow-on group was small, these results may indicate that patients originally not responding to ECT monotherapy can benefit from a more rigorous antidepressant strategy.

In addition, significantly reduced Glx levels were observed for UPs pre-ECT, which increased significantly after successful ECT. Similar results for unipolar depressives were observed for the left anterior cingulate (Pfleiderer et al, 2003), a region intensively interconnected with the amygdala (LeDoux, 2003). This finding indicates a pertubated Glx metabolism, which is dependent on intact neuron and/or glial cell function. Consequently, ECT may also contribute to increased NAA levels because of improved glial cell function and glutamate metabolism.

Interestingly, the analysis of voxel composition, comprising amygdala and about one-third of the anterior hippocampus, revealed an increased GM fraction in patients compared to controls before and also after ECT. Although reports on functional and structural changes of the amygdala related to depressive disorders are conflicting with respect to alterations in size (decrease $v s$ increase) or metabolism (Altshuler et al, 1998; Drevets et al, 1999; Sheline et al, 1998), they nevertheless indicate a possible involvement of the left amygdala in the pathogenesis of 
major mood disorder. The observed positive correlation of GM fraction in our MR voxels with duration of illness may reflect long-term structural alterations occurring with mood disorders. This was also reported in a recent preliminary MRI study of larger left temporal lobes with increasing duration of illness (Ali et al, 2001). Consequently, the amygdalar region may be a suitable region for detecting neurotrophic effects by MRS, since it is a neuroplastic active brain area (LeDoux, 2000) and fills the voxel mainly with GM (Davis and Whalen, 2001). These may well be the neuroanatomic reasons for Ende et al (2000), not having been able to demonstrate an NAA increase after ECT in the bilateral hippocampus.

Our observations are limited by the relatively short offmedication period in this severely ill population. Consequently, pre-ECT pharmacotherapy and/or withdrawal of medication may influence metabolite levels. However, these medications had proved to be ineffective. As expected from an open label and naturalistic design, ECT responders and nonresponders may differ in various uncontrolled aspects (eg medication pre-ECT), which may influence our data. Whether neurotrophic effects may be relevant to antidepressant efficacy should be confirmed in a greater number of patients. Nevertheless our data support previously described neurotrophic effects of ECT in animals (Lamont et al, 2001; Vaidya et al, 1999; Madsen et al, 2000; Parent et al, 1997) and offer initial evidence that this may be important for effective antidepressant therapy in humans.

\section{ACKNOWLEDGEMENTS}

This work was supported by an innovative medical research' (IMF) grant from the University of Münster, Germany (ER 219918)

\section{REFERENCES}

Ali SO, Denicoff KD, Altshuler LL, Hauser P, Li X, Conrad AJ et al (2001). Relationship between prior course of illness and neuroanatomic structures in bipolar disorder. Neuropsychiatry Neurobiological Behav Neurol 14: 227-232.

Altshuler LL, Bartzokis G, Grieder T, Curran J, Mintz J (1998). Amygdala enlargement in bipolar disorder and hippocampal reduction in schizophrenia: an MRI study demonstrating neuroanatomic specificity. Arch Gen Psychiatry 55: 663-664.

Auer DP, Pütz B, Kraft E, Lipinski B, Schill J, Holsboer F (2000). Reduced glutamate in the anterior cingulate cortex in depression: an in vivo proton magnetic resonance spectroscopy study. Biol Psychiatry 47: 305-313.

Baslow MH (2000). Functions of $N$-acetyl-L-aspartate and $N$-acteylL-aspartylglutamate in the vertebrate brain: role in glial cellspecific signaling. J Neurochem 75: 453-459.

Bhakoo KK, Pearce D (2000). In vitro expression of $N$-Acetyl asparate by oligodendrocytes: implications for proton magnetic resonance spectroscopy signal in vivo. $J$ Neurochem 74: 254-262.

Bonne O, Krausz Y, Shapira B, Bocher M, Karger H, Gorfine M et al (1996). Increased cerebral blood flow in depressed patients responding to electroconvulsing therapy. J Nucl Med 37: 10751080 .
Chen G, Rajkowska G, Du F, Seraji-Bozorgzad N, Manji HK (2000). Enhancement of hippocampal neurogenesis by lithium. $J$ Neurosci 75: 1729-1734.

Davis M, Whalen PG (2001). The amygdala: vigilance and emotion. Mol Psychiatry 6: 13-34.

Drevets WC (2000). Functional anatomical abnormalities in limbic and prefrontal cortical structures in major depression. Prog Brain Res 126: 413-431.

Drevets WC, Gadde KM, Krishnan KRR (1999). Neuroimaging studies of mood disorders. In: DS Charney, EJ Nestler, BS Bunney (eds). Neurobiology of Mental Illness. Oxford University Press: New York, Oxford. pp 394-418.

Duman RS, Heniger GR, Nestler EJ (1997). A molecular and cellular theory of depression. Arch Gen Psychiatry 54: 597-606.

Duman RS, Malberg J, Thome J (1999). Neural plasticity to stress and antidepressant treatment. Biol Psychiatry 46: 1181-1191.

Duman RS, Vaidya VA (1998). Molecular and cellular actions of chronic electroconvulsive seizures. JECT 14: 181-193.

Ende G, Braus DF, Walter S, Weber-Fahr W, Henn FA (2000). The hippocampus in patients treated with electroconvulsive therapy. Arch Gen Psychiatry 57: 937-943.

Fink M (2001). Convulsive therapy: a review of the first 55 years. $J$ Affect Disord 63: 1-15.

Hiller W, Zaudig M, Mombour W (2000). Internationale Diagnosen Checklisten (IDCL) für DSM-IV. Hogrefe Verlag: Göttingen.

Jacobs BL, van Praag H, Gage FH (2000). Adult brain neurogenesis and psychiatry: a novel theory of depression. Mol Psychiatry 5: 262-269.

Lamont SR, Pauls A, Stewart CA (2001). Repeated electroconvulsive stimulation, but not antidepressant drugs, induces mossy fibre sprouting in the rat hippocampus. Brain Res 893: 53-58.

LeDoux JE (2000). Emotion circuits in the brain. Ann Rev Neurosci 23: $155-184$.

Madsen AL, Keiding N, Karle A, Esbjerg S, Hemmingsen R (1998). Neuroleptics in progressive structural brain abnormalities in psychiatric illness. Lancet 352: 784-785.

Madsen TM, Treschow A, Bengzon J, Bolwig TG, Lindvall O, Tingström A (2000). Increased neurogenesis in a model of electroconvulsive therapy. Biol Psychiatry 47: 1043-1049.

Manji HK, Moore GJ, Rajkowska G, Chen G (2000). Neuroplasticity and cellular resilience in mood disorders. Mol Psychiatry 5: 578593.

Moore GJ, Bebchuk JM, Hasanat K, Chen G, Serji-Bozorgzad N, Wilds IB et al (2000). Lithium increases $\mathrm{N}$-acetyl-aspartate in the human brain: in vivo evidence in support of bcl-2's neurotrophic effects? Biol Psychiatry 48: 1-8.

Parent JM, Yu TW, Leibowitz RT, Geschwind DH, Sloviter RS, Lowenstein DH (1997). Dentate granule cell neurogenesis is increased by seizures and contributes to aberrant network reorganization in the adult hippocampus. J Neurosci 17: 37273738.

Pfleiderer B, Michael N, Erfurth A, Ohrmann P, Homann U, Wolgast $\mathrm{M}$ et al (2003). Effective electroconvulsive therapy reverses glutamate/glutamine deficit in the left anterior cingulum of unipolar depressed patients. J Psychiat Res: Neuroimag, in press.

Provencher SW (1993). Estimation of metabolite concentrations from localized in vivo proton NMR spectra. Mag Res Med 30: 672-679.

Prudic J, Haskett RF, Mulsant B, Malone KM, Pettinati HM, Stephens $S$ et al (1996). Resistance to antidepressant medications and short-term clinical response to ECT. Am J Psychiatry 153: 985-992.

Sager TN, Topp S, Torup L, Hanson LG, Egestod B, Moller A (2001). Evaluation of CA1 damage using single-voxel ${ }^{1} \mathrm{H}-\mathrm{MRS}$ and un-biased stereology: can non-invasive measures of 
$\mathrm{N}$-acetyl-aspartate following global ischemia be used as a reliable measure of neuronal damage? Brain Res 892: 166-175.

Sanacora G, Mason GF, Rothman DL, Behar KL, Hyder F, Petroff OA et al (1999). Reduced cortical g-aminobutyric acid levels in depressed patients determined by proton magnetic spectroscopy. Arch Gen Psychiatry 56: 1043-1047.

Sheline YI, Gado MH, Price JL (1998). Amygdala core nuclei volumes are decreased in recurrent major depression. Neuroreport 9: 2023-2028.

Simmons ML, Frondoza CG, Coyle JT (1991). Immunocytochemical localization of $\mathrm{N}$-acetyl-aspartate with monoclonal antibodies. Neuroscience 45: 37-45.
Tsai G, Passani LA, Slusher BS, Carter R, Kleinman JE, Coyle JT (1995). Abnormal excitatory neurotransmitter metabolism in schizophrenic brains. Arch Gen Psychiatry 52: 829-836.

Vaidya VA, Siuciak JA, Du F, Duman RS (1999). Hippocampal mossy fiber sprouting induced by chronic electroconvulsive seizures. Neuroscience 89: 157-166.

Wang Y, Li SJ (1998). Differentiation of metabolic concentrations between grey matter and white matter of human brain by in vivo ${ }^{1} \mathrm{H}$ magnetic resonance spectroscopy. Mag Res Med 39: $28-33$. 